\title{
Immune response in melanoma: an in-depth analysis of the primary tumor and corresponding sentinel lymph node
}

Michelle W Ma ${ }^{1,2, *}$, Ratna C Medicherla, ${ }^{1,2, *}$, Meng Qian ${ }^{3}$, Eleazar Vega-Saenz de Miera ${ }^{1,2}$, Erica B Friedman ${ }^{2,4}$, Russell S Berman ${ }^{2,4,5}$, Richard L Shapiro ${ }^{2,4,5}$, Anna C Pavlick ${ }^{1,2,5,6}$, Patrick A Ott ${ }^{2,5,6}$, Nina Bhardwaj ${ }^{1,2,5,6,7}$, Yongzhao Shao ${ }^{2,3}$, Iman Osman ${ }^{1,2,5}$ and Farbod Darvishian ${ }^{2,7}$

${ }^{1}$ Ronald O. Perelman Department of Dermatology, New York University School of Medicine, New York, NY, USA; ${ }^{2}$ Interdisciplinary Melanoma Cooperative Group, New York University School of Medicine, New York, NY, USA; ${ }^{3}$ Division of Biostatistics, New York University School of Medicine, New York, NY, USA; ${ }^{4}$ Department of Surgery, New York University School of Medicine, New York, NY, USA; ${ }^{5}$ New York University Cancer Institute, New York, NY, USA; ${ }^{6}$ Department of Medicine, New York University School of Medicine, New York, NY, USA and ${ }^{7}$ Department of Pathology, New York University School of Medicine, New York, $N Y, U S A$

The sentinel lymph node is the initial site of metastasis. Downregulation of antitumor immunity has a role in nodal progression. Our objective was to investigate the relationship between immune modulation and sentinel lymph node positivity, correlating it with outcome in melanoma patients. Lymph node/primary tissues from melanoma patients prospectively accrued and followed at New York University Medical Center were evaluated for the presence of regulatory T cells $\left(\mathrm{Foxp3}^{+}\right.$) and dendritic cells (conventional: CD11c ${ }^{+}$, mature: $\mathrm{CD} \mathrm{Cb}^{+}$) using immunohistochemistry. Primary melanoma immune cell profiles from sentinel lymph node-positive/negative patients were compared. Logistic regression models inclusive of standard-of-care/immunological primary tumor characteristics were constructed to predict the risk of sentinel lymph node positivity. Immunological responses in the positive sentinel lymph node were also compared with those in the negative non-sentinel node from the same nodal basin and matched negative sentinel lymph node. Decreased immune response was defined as increased regulatory $\mathrm{T}$ cells or decreased dendritic cells. Associations between the expression of these immune modulators, clinicopathological variables, and clinical outcome were evaluated using univariate/multivariate analyses. Primary tumor conventional dendritic cells and regression were protective against sentinel lymph node metastasis (odds ratio $=0.714,0.067 ; P=0.0099,0.0816$, respectively). Antitumor immunity was downregulated in the positive sentinel lymph node with an increase in regulatory $T$ cells compared with the negative non-sentinel node from the same nodal basin $(P=0.0005)$ and matched negative sentinel lymph node $(P=0.0002)$. The positive sentinel lymph node also had decreased numbers of conventional dendritic cells compared with the negative sentinel lymph node $(P<0.0001)$. Adding sentinel lymph node regulatory $T$ cell expression improved the discriminative power of a recurrence risk assessment model using clinical stage. Primary tumor regression was associated with prolonged disease-free $(P=0.025)$ and melanoma-specific $(P=0.014)$ survival. Our results support an assessment of local immune profiles in both the primary tumor and sentinel lymph node to help guide therapeutic decisions.

Modern Pathology (2012) 25, 1000-1010; doi:10.1038/modpathol.2012.43; published online 16 March 2012

Keywords: dendritic cells; lymphatic metastases; melanoma; regulatory T cells; sentinel lymph node biopsy

Correspondence: Dr F Darvishian, MD, Department of Pathology, New York University School of Medicine, 530 First Avenue, Skirball 7N, New York, NY 10016, USA.

E-mail: Farbod.Darvishian@nyumc.org

*These authors contributed equally to this work.

This work was presented in part at the Annual Meeting of the American Society of Clinical Oncology, 3-7 June 2011, Chicago, IL.

Received 12 December 2011; revised 25 January 2012; accepted 25 January 2012; published online 16 March 2012 
Sentinel lymph node status continues to be the most important prognostic factor in melanoma not only for recurrence ${ }^{1}$ but also overall survival. ${ }^{2,3}$ Melanoma patients with thick $(>1.00 \mathrm{~mm})$ and ulcerated primary tumors, in particular, are at high risk for occult nodal metastasis and warrant a sentinel lymph node biopsy to evaluate for the presence of nodal disease. ${ }^{2}$ Sentinel lymph node metastasis, however, does occur in patients with thin $(\leq 1.00 \mathrm{~mm})$ melanomas ${ }^{4}$ such that additional selection criteria may improve the sentinel lymph node positivity risk-stratification model based on thickness and ulceration alone.

Immune cell populations in the primary tumor reflect the host immune response, and there is evidence to suggest that the immunophenotype of this immune response is predictive of sentinel lymph node status not only in melanoma ${ }^{3,5-10}$ but also in colorectal, ${ }^{11}$ esophageal, ${ }^{12}$ gastric, ${ }^{13}$ and papillary thyroid cancer. ${ }^{14}$ Primary tumor-infiltrating lymphocytes have an important role in the antitumor $\mathrm{T}$ cell response that mediates regression, and the absence of tumor-infiltrating lymphocytes and regression have both been shown to predict sentinel lymph node positivity in melanoma. ${ }^{3,5-10}$ The prognostic relevance of different primary tumor $\mathrm{T}$ cell subsets, however, has not yet been examined in melanoma even though studies in other cancers have demonstrated the clinical value of characterizing tumor-infiltrating lymphocyte subpopulations, such as the immunosuppressive Foxp $3^{+}$regulatory $\mathrm{T}$ cell subset, in predicting nodal metastasis. ${ }^{11-14} \mathrm{~T}$ cell responses, furthermore, require the antigen-presenting capacity of dendritic cells, including the immunogenic $\mathrm{CD} 11 \mathrm{c}^{+}$conventional dendritic cells and the tolerogenic CD123 ${ }^{+}$ plasmacytoid dendritic cells. Dendritic cell subsets may therefore also predict sentinel lymph node positivity in melanoma, and one study in melanoma has already shown an association between primary tumor $\mathrm{CD}_{123^{+}}$plasmacytoid dendritic cells and clinical outcome. ${ }^{15}$

Primary melanoma immune markers warrant further investigation as potential predictors of sentinel lymph node status, and given the impact of primary tumor-derived cytokines on the immunological status of the sentinel lymph node ${ }^{16}$ and the importance of the pathological status of the sentinel lymph node, ${ }^{1-3}$ additional prognostic information may be gained by an immunological characterization of the sentinel lymph node as well. In this study, our objective was to first examine the relationship between the immune profile of the primary melanoma and sentinel lymph node positivity and then to compare the immunophenotype of the immune response in positive sentinel lymph nodes with that in negative sentinel lymph nodes and negative non-sentinel nodes from the same nodal basin and to correlate it with clinical outcome in a cohort of prospectively-accrued cutaneous melanoma patients.

\section{Patients and methods}

\section{Study Population}

Lymph node and primary melanoma tissues were retrieved from patients enrolled in the Interdisciplinary Melanoma Cooperative Group (IRB\#10362), ${ }^{17}$ a prospectively collected clinicopathological-biospecimen database at New York University Langone Medical Center (August 2002 to September 2009). Informed consent was obtained from patients at the time of enrollment, and all demographic, clinicopathological, and follow-up data were recorded prospectively.

Patients with available sentinel lymph node biopsy specimens were identified, and sentinel lymph nodepositive patients were matched to sentinel lymph node-negative patients for age at initial melanoma diagnosis, gender, primary tumor thickness $(\mathrm{mm})$, and ulceration status. Other clinicopathological features collected included primary tumor mitotic rate $\left(\right.$ mitoses $/ \mathrm{mm}^{2}$ ), histological subtype, anatomic site, and American Joint Committee on Cancer stage at pathological diagnosis. Negative non-sentinel node tissues were obtained from all sentinel lymph nodepositive patients as well from the same nodal basin as the positive sentinel lymph node.

\section{Assessment of Foxp3, CD11c, and CD86 Expression}

Immunohistochemistry was performed using mouse anti-human Foxp3 clone 236A/E7 (eBioscience, San Diego, CA, USA), CD11c clone 5D11 (Novocastra Laboratories, Newcastle upon Tyne, UK) and CD86 (R\&D Systems, Minneapolis, MN, USA) on formalin fixed, paraffin embedded lymph node and primary melanoma tissues to detect regulatory $\mathrm{T}$ cells $\left(\right.$ Foxp $\left.^{+}\right)$, conventional dendritic cells $\left(\mathrm{CD} 11 \mathrm{c}^{+}\right)$, and mature dendritic cells $\left(\mathrm{CD}^{+} 6^{+}\right)$, respectively. Anti-human CD123 clone BR4MS (Novocastra) was also used to identify plasmacytoid dendritic cells in a subset of sentinel lymph node tissues. In brief, after deparaffinization and rehydration, heatinduced epitope retrieval was performed in $0.01 \mathrm{M}$ citrate buffer (pH 6.0) for Foxp3, CD11c, and CD86 in a $1200-\mathrm{W}$ microwave oven at $100 \%$ power for 20 min. Sections were then cooled in tap water for $5 \mathrm{~min}$, quenched in hydrogen peroxide for $30 \mathrm{~min}$, washed with PBS, and incubated with blocking serum (VECTASTAIN Elite ABC Kit-Mouse IgG, Vector Laboratories, Burlingame, CA, USA) for $30 \mathrm{~min}$ followed by each primary antibody diluted in buffer (Foxp3, 1:500; CD11c, 1:50; CD86, 1:400) at room temperature for $1 \mathrm{~h}$ and at $4^{\circ} \mathrm{C}$ overnight. Slides were washed in buffer and incubated with diluted biotinylated secondary antibodies (horse anti-mouse at 1:500 for both Foxp3- and CD11c-stained sections; horse anti-goat at 1:100 for CD86-stained sections, Vector Laboratories) for $1 \mathrm{~h}$. Avidin-biotinylated horseradish peroxidase complexes diluted at 1:500 (ABC Reagent, Vector 
Laboratories) were added, and complexes were visualized with diaminobenzidine (DAB substrate kit, Vector Laboratories). Slides were then washed in distilled water, counterstained with hematoxylin, dehydrated, and mounted with permanent media. Appropriate positive and negative controls were included with the study sections.

An attending pathologist (FD) who was blinded to patients' clinical data scored Foxp3, CD11c, CD86, and CD123 expression as the absolute number of positively-stained immune cells demonstrating characteristic $\mathrm{T}$ cell and dendritic cell morphology, respectively, in a representative high-power field (HPF; $0.2 \mathrm{~mm}^{2}$ ) that was selected by scanning each slide at $\times 40$ to find the field with the highest antibody expression. Tumor involvement in each specimen was also assessed semi-quantitatively as the percentage of all cells using the corresponding hematoxylin and eosin stained-section.

\section{Statistical Analysis}

Descriptive statistics were used to summarize demographic and primary tumor characteristics of melanoma patients. Distributional comparison of continuous variables between the sentinel lymph node-positive and sentinel lymph node-negative groups was made using the two-sided WilcoxonMann-Whitney test. Distributional comparison of continuous variables between the positive sentinel lymph node and negative non-sentinel node from the same nodal basin was made using the paired $t$-test for variables that approximately follow the normal distribution. The $\chi^{2}$ test, Fisher's exact test, and Armitage trend test were used to compare independent proportions for categorical variables. Logistic regression models were used to assess the significance of predictors and to calculate the odds ratios with or without adjustments of other covariates/factors. The area under the receiver operating characteristic curve was calculated as an indication of the discriminative power of the logistic predictive models. Statistical significance of tests was claimed when $P$-values were $<5 \% \quad(P<0.05)$. In survival analysis, Kaplan-Meier curves and log-rank tests were used to assess the differential survival profiles of the low- and high-risk groups. Statistical analyses were conducted using SAS and the statistical software $R$.

\section{Results}

Sentinel lymph node tissue was available for analysis from 84 melanoma patients. In all, 31 sentinel lymph node-positive patients were matched by age at pathological diagnosis, gender, primary tumor thickness, and ulceration status to 53 sentinel lymph node-negative patients. Primary tumor thickness and ulceration status were similar between the two groups (median thickness: $2.50 \mathrm{~mm} \mathrm{vs} 2.20 \mathrm{~mm}$,
$P=0.750$; ulceration present: $35 \%$ vs $36 \%, P=0.923$, respectively; Table 1). There was no significant difference in age at initial melanoma diagnosis or gender as well primary tumor mitotic rate, histological subtype, and anatomic site between sentinel lymph node-positive and -negative patients $(P>0.05$; Table 1$)$. Twenty-three patients recurred during follow-up (median: 3.6 years for the whole cohort, 3.8 years for survivors): 13/31 (42\%) sentinel lymph nodepositive patients and 10/53 (19\%) sentinel lymph node-negative patients.

In all, 54 (64\%) of the 84 patients also had primary melanoma specimens available for analysis: 23 sentinel lymph node-positive and 31 sentinel lymph node-negative patients. Median primary tumor thickness was similar between sentinel lymph node-positive and -negative patients (2.25 mm vs $2.30 \mathrm{~mm}, P=0.92$; Table 1). There were also no significant differences between these two groups in age at pathological diagnosis, gender, primary tumor ulceration status, mitotic rate, histological subtype, or anatomic site $(P>0.05$; Table 1). Sentinel lymph node-positive patients did, however, have primary tumors with a significantly higher tumor volume (mean: 63\% vs 46\%, $P=0.043$ ) and a lower number of CD11c ${ }^{+}$conventional dendritic cells/HPF (mean: 9 vs 16, $P=0.054$ ) compared with those from sentinel lymph nodenegative patients as well as a decreased proportion of primaries with regression ( $9 \%$ vs $35 \%, P=0.028$ ) (Table 2). Yet, no significant difference in the presence of tumor-infiltrating lymphocytes, the number of regulatory $\mathrm{T}$ cells or mature dendritic cells/HPF, or solar elastosis were observed between the two groups $(P>0.05$; Table 2$)$.

\section{The Immune Profile of Primary Melanomas Predicts Sentinel Lymph Node Positivity}

Three different logistic regression models for predicting sentinel lymph node positivity are shown in Table 3. Model 1 includes primary tumor thickness and ulceration status, but as neither covariate independently predicts sentinel lymph node status, this model has a limited discriminatory accuracy with an area under the receiver operating characteristic curve equal to 0.5520 (Figure 1a). Model 2 is based solely on the immune profile of the primary melanoma (tumor-infiltrating lymphocytes, Foxp3 ${ }^{+}$ regulatory $\mathrm{T}$ cells, $\mathrm{CD} 11 \mathrm{c}^{+}$conventional dendritic cells, $\mathrm{CD}^{+} 6^{+}$mature dendritic cells, regression, and solar elastosis), and its discriminatory accuracy is better with an area under the curve equal to 0.8205 (Figure 1b). Both primary tumor conventional dendritic cells and regression are protective against lymph node metastasis, but only conventional dendritic cells are significantly associated with sentinel lymph node status (odds ratio $=0.853$, 0.158; $P=0.0092$, 0.0698, respectively). Model 3 then combines standard-of-care primary tumor 
Table 1 Demographic and primary melanoma features of patients who underwent a sentinel lymph node biopsy

\begin{tabular}{|c|c|c|c|c|c|c|}
\hline & \multicolumn{3}{|c|}{ Sentinel lymph node tissue available } & \multicolumn{3}{|c|}{ Primary tumor tissue available } \\
\hline & $\begin{array}{c}\text { Sentinel lymph } \\
\text { node-positive } \\
\mathrm{N}=31\end{array}$ & $\begin{array}{l}\text { Sentinel lymph } \\
\text { node-negative } \\
\mathrm{N}=53\end{array}$ & $\mathrm{P}$-value & $\begin{array}{c}\text { Sentinel lymph } \\
\text { node-positive } \\
\mathrm{N}=23\end{array}$ & $\begin{array}{c}\text { Sentinel lymph } \\
\text { node-negative } \\
\mathrm{N}=31\end{array}$ & $\mathrm{P}$-value \\
\hline Age at pathological diagnosis (years) & & & 0.145 & & & 0.21 \\
\hline Median (range) & $54(28-79)$ & $65(29-86)$ & & $62(28-79)$ & $64(34-84)$ & \\
\hline Gender & & & 0.358 & & & 0.44 \\
\hline Male, $n(\%)$ & $19(61)$ & $27(51)$ & & $15(65)$ & $17(55)$ & \\
\hline Female, $n(\%)$ & $12(39)$ & $26(49)$ & & $8(35)$ & $14(45)$ & \\
\hline Primary tumor thickness (mm) & & & 0.750 & & & 0.92 \\
\hline Median (range) & $2.50(0.90-20)$ & $2.20(0.80-12)$ & & $2.25(0.90-20)$ & $2.30(0.80-12)$ & \\
\hline Primary tumor ulceration status & & & 0.923 & & & 0.38 \\
\hline Absent, $n(\%)$ & $20(65)$ & $34(64)$ & & $16(70)$ & $18(58)$ & \\
\hline Present, $n(\%)$ & $11(35)$ & $19(36)$ & & $7(30)$ & $13(42)$ & \\
\hline Primary tumor mitotic rate (mitoses $/ \mathrm{mm}^{2}$ ) & & & 0.14 & & & $0.38^{\mathrm{b}}$ \\
\hline $0, n(\%)$ & $1(3)$ & $8(15)$ & & $1(4)$ & $4(13)$ & \\
\hline$\geq 1, n(\%)$ & $29(94)$ & $44(83)$ & & $22(96)$ & $27(87)$ & \\
\hline Unclassified, $n(\%)$ & $1(3)$ & $1(2)$ & & $0(0)$ & $0(0)$ & \\
\hline AJCC stage at pathological diagnosis & & & $<0.0001$ & & & $<0.0001$ \\
\hline $\mathrm{I}, n(\%)$ & $0(0)$ & $21(40)$ & & $0(0)$ & $11(35)$ & \\
\hline II, $n(\%)$ & $0(0)$ & $31(58)$ & & $0(0)$ & $20(65)$ & \\
\hline III, $n(\%)$ & $31(100)$ & $1(2)^{a}$ & & $23(100)$ & $0(0)$ & \\
\hline Primary tumor histological subtype & & & 0.146 & & & $1^{\mathrm{b}}$ \\
\hline Superficial spreading melanoma, $n(\%)$ & $9(29)$ & $19(36)$ & & $8(35)$ & $10(32)$ & \\
\hline Nodular melanoma, $n(\%)$ & $17(55)$ & $28(53)$ & & $12(52)$ & $17(55)$ & \\
\hline Other melanoma, $n(\%)$ & $4(13)$ & $5(9)$ & & $3(13)$ & $4(13)$ & \\
\hline Unclassified, $n(\%)$ & $1(3)$ & $1(2)$ & & $0(0)$ & $0(0)$ & \\
\hline Primary tumor anatomic site & & & 0.280 & & & $0.062^{\mathrm{b}}$ \\
\hline Head/neck, $n(\%)$ & $4(13)$ & $3(6)$ & & $4(17)$ & $3(10)$ & \\
\hline Axial, $n(\%)$ & $10(32)$ & $25(47)$ & & $5(22)$ & $17(55)$ & \\
\hline Extremity, $n(\%)$ & $17(55)$ & $25(47)$ & & $14(61)$ & $11(35)$ & \\
\hline
\end{tabular}

Abbreviation: AJCC, American Joint Committee on Cancer.

Characteristics for the entire cohort $(N=84)$ and the subset with available primary tissue for analysis $(N=54)$ are listed separately.

${ }^{\mathrm{a}}$ Satellites without metastatic nodes.

${ }^{\mathrm{b}}$ By Fisher's exact test.

characteristics and markers of the immune response to achieve an even higher discriminatory accuracy with an area under the curve equal to 0.9158 (Figure 1c).

Primary tumor conventional dendritic cells continue to be protective (odds ratio $=0.714$,

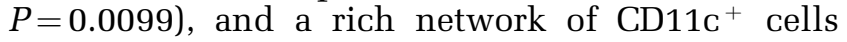
can be seen in the primary melanoma from a sentinel lymph node-negative patient (Figures 1d-f) in contrast to the few CD11c ${ }^{+}$cells present in the primary tumor from a sentinel lymph node-positive patient (Figures 1g-i). Regression remains protective against lymph node progression (odds ratio $=0.067$ ) as well but only trends towards significance $(P=0.0816)$ as in the previous model. Increasing age at pathological diagnosis is also protective (odds ratio $=0.942, P=0.0548$ ), whereas a higher tumor volume, the presence of tumor-infiltrating lymphocytes, and an increased proportion of mature dendritic cells are all risk factors for sentinel lymph node positivity (odds ratio $=1.031,10.790,1.350$; $P=0.0546,0.0451,0.0330$, respectively). There is no concordance, however, between the immune profile of the primary melanoma and the sentinel lymph node (Supplementary Table 1).

\section{The Immunologic Balance in the Positive Sentinel Lymph Node Is Shifted Towards Tolerance}

Positive sentinel lymph nodes have a tolerogenic immune profile compared with negative sentinel lymph nodes with the latter containing significantly fewer immunosuppressive $\mathrm{Foxp}^{+}$regulatory T cells/HPF (median: 45 vs 80, $P=0.0002$; Figures 2a-c) and more immunogenic CD11 ${ }^{+}$conventional dendritic cells/HPF (median: 35 vs 20, $P=0.00002$; Figures $2 \mathrm{~d}-\mathrm{f}$ ). Yet, the number of CD86 ${ }^{+}$ 
Table 2 Immune profile of the primary melanoma in sentinel lymph node-positive vs sentinel lymph node-negative patients

\begin{tabular}{|c|c|c|c|}
\hline & $\begin{array}{l}\text { Sentinel lymph } \\
\text { node-positive } \\
\mathrm{N}=23\end{array}$ & $\begin{array}{l}\text { Sentinel lymph } \\
\text { node-negative } \\
\mathrm{N}=31\end{array}$ & $\mathrm{P}$-value \\
\hline $\begin{array}{l}\text { Percentage of tumor cells in primary (\%) } \\
\text { Mean; median }\end{array}$ & $63 ; 70$ & $46 ; 40$ & 0.043 \\
\hline $\begin{array}{l}\text { Primary tumor-infiltrating lymphocytes } \\
\text { Absent, } n(\%) \\
\text { Present, } n(\%)\end{array}$ & $\begin{array}{r}6(26) \\
17(74)\end{array}$ & $\begin{array}{r}9(29) \\
22(71)\end{array}$ & 0.85 \\
\hline $\begin{array}{l}\text { Primary tumor Foxp } 3^{+} \text {regulatory T cells (\#/HPF) } \\
\text { Mean; median }\end{array}$ & $41 ; 35$ & $44 ; 35$ & 0.87 \\
\hline $\begin{array}{l}\text { Primary tumor } C D 11 C^{+} \text {conventional dendritic cells (\#/HPF) } \\
\quad \text { Mean; median }\end{array}$ & $9 ; 8$ & $16 ; 12$ & 0.054 \\
\hline $\begin{array}{l}\text { Primary tumor } C D 86^{+} \text {mature dendritic cells (\#/HPF) } \\
\quad \text { Mean; median }\end{array}$ & $5 ; 4$ & $4 ; 3$ & 0.12 \\
\hline $\begin{array}{l}\text { Primary tumor regression } \\
\text { Absent, } n(\%) \\
\text { Present, } n(\%)\end{array}$ & $\begin{aligned} 21 & (91) \\
2 & (9)\end{aligned}$ & $\begin{array}{l}20(65) \\
11(35)\end{array}$ & $0.028^{\mathrm{a}}$ \\
\hline $\begin{array}{l}\text { Primary tumor solar elastosis } \\
\text { Absent, } n(\%) \\
\text { Present, } n(\%)\end{array}$ & $\begin{array}{r}16(70) \\
7(30)\end{array}$ & $\begin{array}{l}16(52) \\
15(48)\end{array}$ & 0.18 \\
\hline
\end{tabular}

Abbreviation: HPF, high-power field.

${ }^{\mathrm{a}}$ By Fisher's exact test.

Table 3 Logistic regression models for predicting sentinel lymph node positivity in melanoma patients

\begin{tabular}{|c|c|c|c|c|}
\hline Model & Covariates & Odds ratio & $95 \%$ Confidence interval & $\mathrm{P}$-value \\
\hline 1 & $\begin{array}{l}\text { Primary tumor thickness (mm) } \\
\text { Primary tumor ulceration (present vs absent) }\end{array}$ & $\begin{array}{l}1.076 \\
0.801\end{array}$ & $\begin{array}{l}0.927-1.248 \\
0.288-2.228\end{array}$ & $\begin{array}{l}0.3355 \\
0.6709\end{array}$ \\
\hline 2 & $\begin{array}{l}\text { Primary tumor-infiltrating lymphocytes (present vs absent) } \\
\text { Primary tumor Foxp3 } 3^{+} \text {regulatory T cells (\#/HPF) } \\
\text { Primary tumor CD } 11 \mathrm{c}^{+} \text {conventional dendritic cells (\#/HPF) } \\
\text { Primary tumor CD86 }{ }^{+} \text {mature dendritic cells (\#/HPF) } \\
\text { Primary tumor regression (present vs absent) } \\
\text { Primary tumor solar elastosis (present vs absent) }\end{array}$ & $\begin{array}{l}2.787 \\
1.004 \\
0.853 \\
1.133 \\
0.158 \\
0.852\end{array}$ & $\begin{array}{l}0.530-14.648 \\
0.980-1.030 \\
0.756-0.961 \\
0.970-1.324 \\
0.022-1.161 \\
0.215-3.372\end{array}$ & $\begin{array}{l}0.2261 \\
0.7276 \\
\mathbf{0 . 0 0 9 2} \\
0.1143 \\
0.0698 \\
0.8190\end{array}$ \\
\hline 3 & $\begin{array}{l}\text { Primary tumor thickness (mm) } \\
\text { Primary tumor ulceration (present vs absent) } \\
\text { Percentage of tumor cells in primary (\%) } \\
\text { Primary tumor-infiltrating lymphocytes (present vs absent) } \\
\text { Primary tumor CD } 11 \mathrm{c}^{+} \text {conventional dendritic cells (\#/HPF) } \\
\text { Primary tumor CD86 }{ }^{+} \text {mature dendritic cells (\#/HPF) } \\
\text { Primary tumor regression (present vs absent) } \\
\text { Age at pathological diagnosis (years) }\end{array}$ & $\begin{array}{r}1.245 \\
0.151 \\
1.031 \\
10.790 \\
0.714 \\
1.350 \\
0.067 \\
0.942\end{array}$ & $\begin{array}{l}0.834-1.860 \\
0.016-1.457 \\
0.999-1.064 \\
1.053-110.552 \\
0.553-0.923 \\
1.024-1.779 \\
0.003-1.402 \\
0.886-1.001\end{array}$ & $\begin{array}{l}0.2831 \\
0.1021 \\
\mathbf{0 . 0 5 4 6} \\
\mathbf{0 . 0 4 5 1} \\
\mathbf{0 . 0 0 9 9} \\
\mathbf{0 . 0 3 3 0} \\
0.0816 \\
\mathbf{0 . 0 5 4 8}\end{array}$ \\
\hline
\end{tabular}

Abbreviation: HPF, high-power field.

Bold values are significant $P$-values.

mature dendritic cells is significantly decreased in the negative sentinel lymph node compared with the positive sentinel lymph node (median: $20 \mathrm{vs} 36$, $P=0.0005$; Figures 2g-i). A subset analysis of those sentinel lymph node-positive cases with the highest number of $\mathrm{CD}^{+}{ }^{+}$mature dendritic cells, however, showed that the immunosuppressive CD $123^{+}$ plasmacytoid dendritic cell subset exceeded the immunogenic $\mathrm{CD}_{11 \mathrm{C}}{ }^{+}$conventional dendritic cell subset by up to five fold (Supplementary Figure 1). The immunological balance in the positive sentinel lymph node compared with that in the negative nonsentinel node from the same nodal basin is similarly shifted away from an antitumor immune response with its increased number of $\mathrm{Foxp}^{+}$regulatory $\mathrm{T}$ cells $(P=0.0005$; Figures $3 \mathrm{a}, \mathrm{b})$ and decreased number of $\mathrm{CD}_{11 \mathrm{C}^{+}}$conventional dendritic cells $\left(P=0.059\right.$; Figures 3c, d). $\mathrm{CD}^{+} 6^{+}$mature dendritic cells are likewise increased in the positive sentinel lymph node compared with the negative nonsentinel node from the same nodal basin $(P=0.06$; Figures 3e, f) as in the previous comparison. 

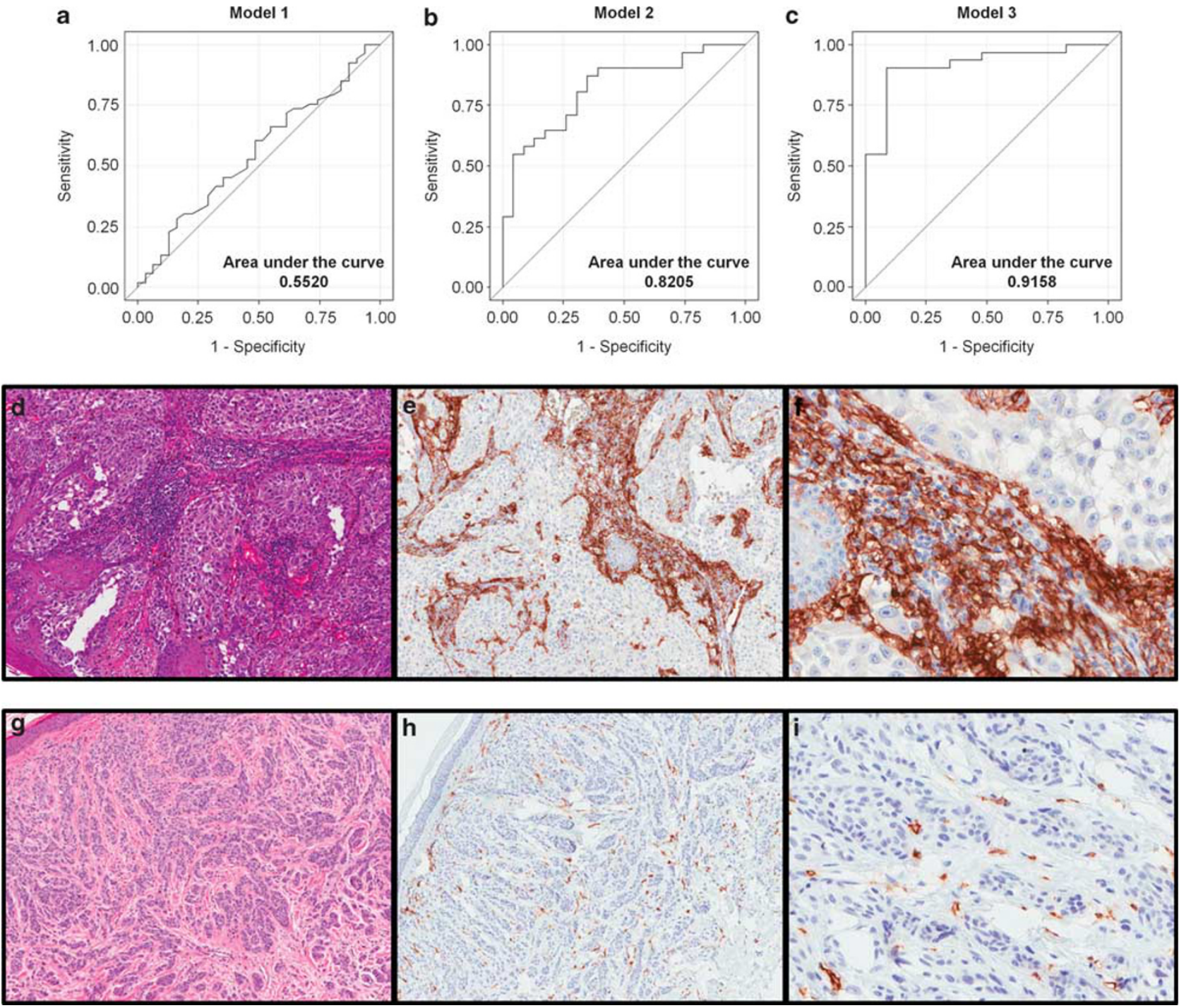

Figure 1 Immune profile of primary melanomas predicts sentinel lymph node positivity. Receiver operating characteristic curves for three risk stratification models to predict sentinel lymph node positivity in melanoma patients: (a) Model 1, (b) Model 2, and (c) Model 3. (d) Hematoxylin and eosin-stained primary tissue section $(\times 100)$ from a sentinel lymph node-negative patient whose primary melanoma demonstrates strong immunoreactivity for CD11c $(\times 100$, e). (f) High-power view $(\times 400)$ showing a dense network of $\mathrm{CD}_{11 \mathrm{c}^{+}}$cells exhibiting characteristic dendritic cell morphology. (g) Primary melanoma section stained with hematoxylin and eosin $(\times 100)$ from a patient with a positive sentinel lymph node whose primary tumor illustrates weak CD11c immunostaining $(\times 100, \mathbf{h} ; \times 400, \mathbf{i})$.

\section{Sentinel Lymph Node and Primary Tumor Immune Profiles Contribute to Recurrence Risk}

Sentinel lymph node positivity alone is associated with an increased risk of recurrence (odds ratio $=$ 3.106, $P=0.0250$ ). The addition of sentinel lymph node regulatory T cells to a logistic regression model with clinical stage alone as a predictor of recurrence improves its discriminative power, increasing its area under the receiver operating characteristic curve from 0.6871 to 0.7598 . Primary tumor immune markers contribute to recurrence risk as well, and the presence of regression, in particular, is associated not only with prolonged progression-free survival $(P=0.025$; Figure 4a) but also longer melanoma-specific survival $(P=0.014$; Figure $4 \mathrm{~b})$.

\section{Discussion}

Our study supports the crucial role of the host immune response in both melanoma progression and clinical outcome. Data suggest that the immune profile of the primary melanoma predicts sentinel lymph node positivity and that the presence of primary tumor regression is a favorable prognostic factor in thick $(>2.0 \mathrm{~mm})$ melanoma patients. Modulation of antitumor immunity occurs in the 

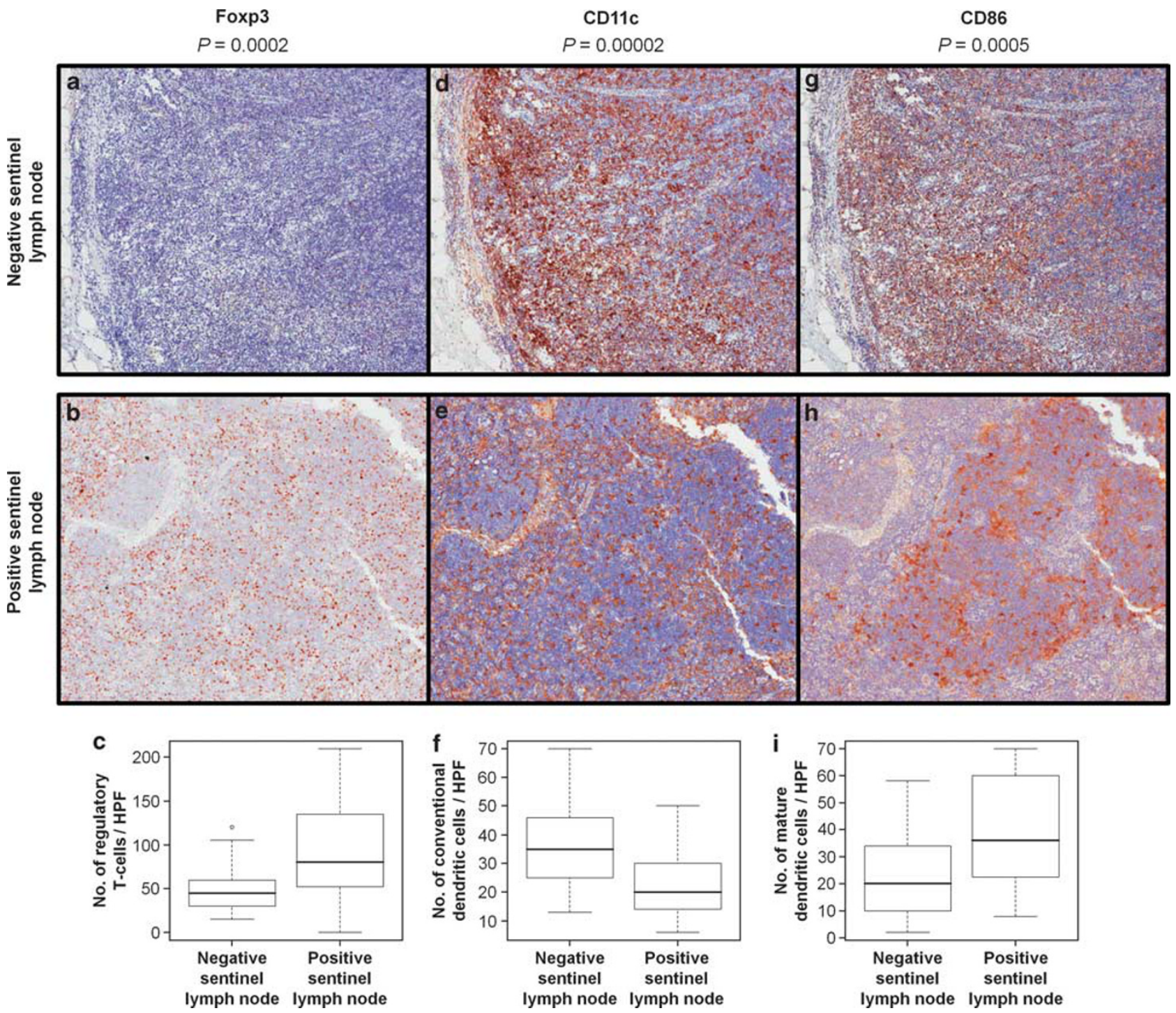

Figure 2 Immunological balance in the positive sentinel lymph node is shifted towards tolerance. Consecutive negative sentinel lymph node and positive sentinel lymph node sections were each stained for Foxp3, CD11c, and CD86. Representative negative sentinel lymph node (a) illustrates weak Foxp3 immunoreactivity compared with the positive sentinel lymph node $(\mathbf{b} ; \times 100)$, and corresponding box plots (c) show a significant increase in the number of Foxp ${ }^{+}$regulatory T cells/HPF in the positive sentinel lymph node. Prominent CD11c immunostaining is shown in the consecutive negative sentinel lymph node section (d) as compared with that in the positive sentinel lymph node $(\mathbf{e} ; \times 100)$, and box plots illustrate this significant decrease in the number of CD11c ${ }^{+}$conventional dendritic cells/ HPF in the positive sentinel lymph node (f). Less intense CD86 immunostaining in the next consecutive negative sentinel lymph node section ( $(\mathrm{g})$ is shown as well in comparison to the increased density of CD86 ${ }^{+}$cells with dendritic cell morphology in the positive sentinel lymph node $(\mathbf{h} ; \times 100)$, a finding also represented in the corresponding box plots $(\mathbf{i})$.

sentinel lymph node as well, and the degree of immunosuppression in the sentinel lymph node microenvironment as measured by the number of Foxp $^{+}$regulatory $\mathrm{T}$ cells, CD11 ${ }^{+}$conventional dendritic cells, and $\mathrm{CD}^{+} 6^{+}$mature dendritic cells, varies with the extent of tumor involvement. Highly tolerogenic microenvironments are associated with advanced disease, and the immunophenotype of the immune response in the sentinel lymph node provides additional, independent prognostic information to determine recurrence risk.

Primary tumor immune markers have previously been shown to predict sentinel lymph node meta- stasis in melanoma, ${ }^{3,5-10}$ and our data provide further evidence in support of the predictive value of primary tumor regression and tumor-infiltrating lymphocytes as it relates to sentinel lymph node positivity. Regression results from a $\mathrm{T}$ cell immune response, and our study suggests that its presence is associated with a decreased risk of nodal progression, which is consistent with data from other melanoma studies. ${ }^{3,5-7}$ Yet, some studies contend that primary tumor regression is a risk factor for sentinel lymph node positivity, albeit in patients with thin $(\leq 1.00 \mathrm{~mm})$ melanomas who are then selected to undergo a sentinel lymph node biopsy. ${ }^{18,19}$ It is 

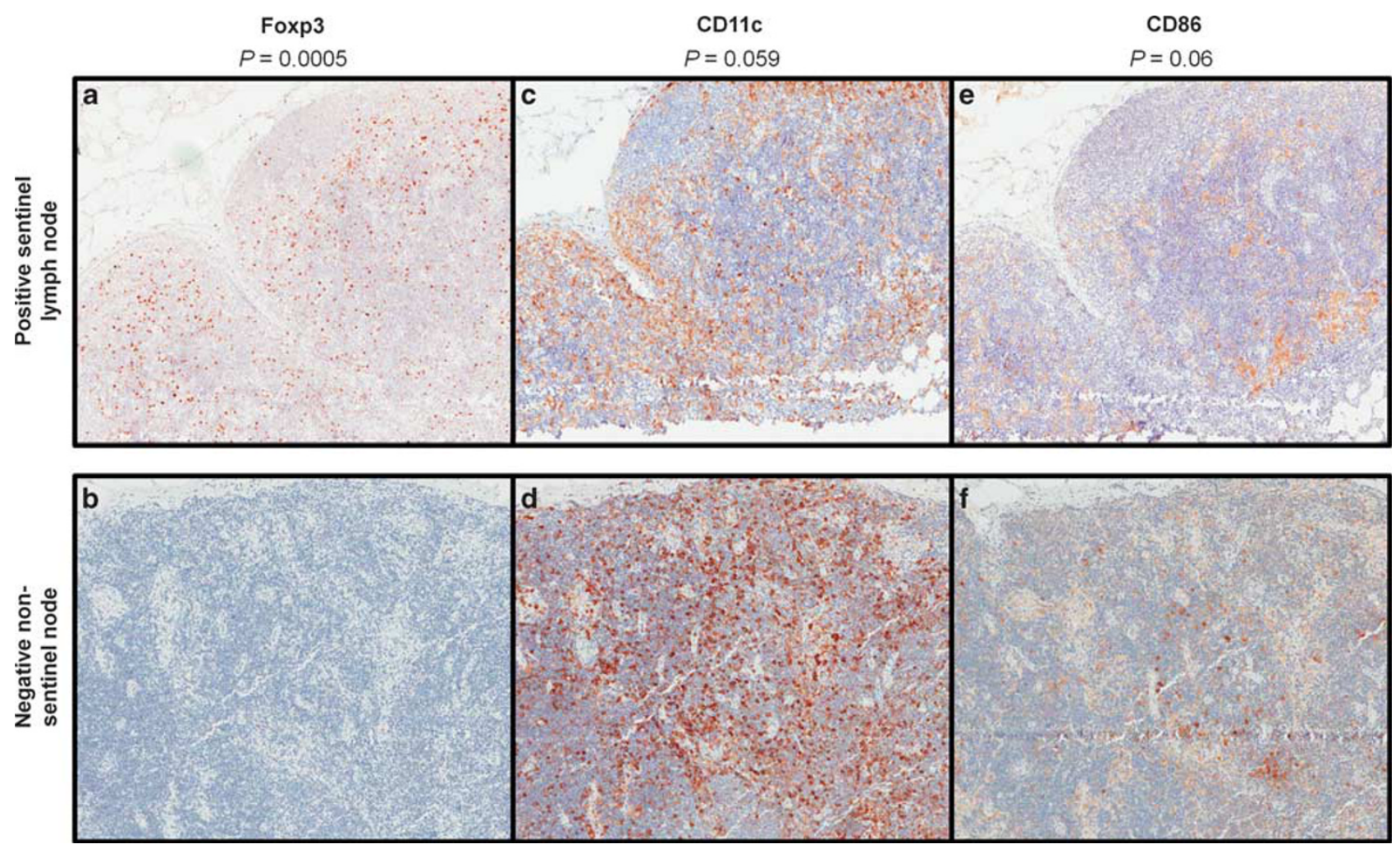

Figure $3 \mathrm{Foxp}^{+}$regulatory $\mathrm{T}$ cells decrease progressively in the positive nodal basin. Consecutive sections from a representative positive sentinel lymph node and a negative non-sentinel node from the same nodal basin were stained for Foxp3, CD11c, and CD86. Strong immunoreactivity for Foxp3 is shown in the positive sentinel lymph node (a) as compared with the negative non-sentinel node (b; $\times$ 100). Lower levels of CD11c immunopositive cells with characteristic dendritic cell morphology are found in the positive sentinel lymph node $(\mathbf{c})$ in comparison to the negative non-sentinel node $(\mathbf{d})(\times 100)$, whereas mildly elevated levels of CD86 expression are seen in the positive sentinel lymph node $(\mathbf{e})$ compared with the negative non-sentinel node $(\mathbf{f} ; \times 100)$.
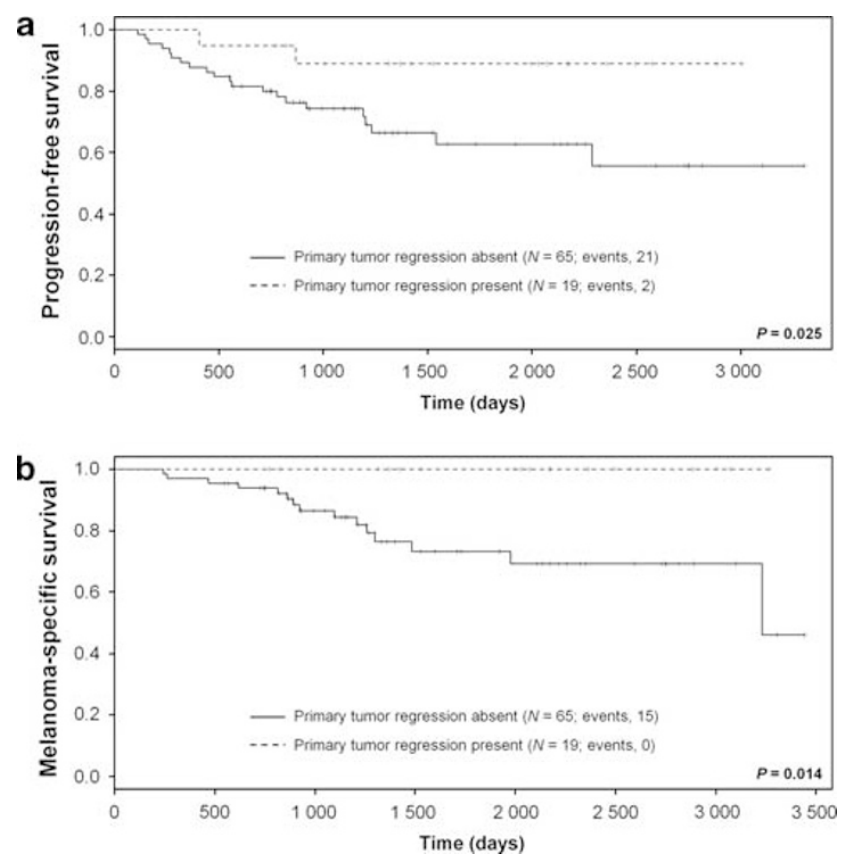

Figure 4 Primary melanoma regression is associated with prolonged survival. Kaplan-Meier estimates of (a) progression-free survival and (b) melanoma-specific survival stratified according to primary tumor regression. important to recognize that our study cohort had thicker melanomas (median: $2.28 \mathrm{~mm}$ ), such that our findings are not disputing the claim that regression in thin melanomas is a poor prognostic factor. ${ }^{18,19}$ There is, however, an apparent contradiction between our study and others regarding the relationship between primary tumor-infiltrating lymphocytes and sentinel lymph node metastasis. Much of the evidence supports the protective role of tumor-infiltrating lymphocytes ${ }^{8-10}$ in contrast to our finding that tumor-infiltrating lymphocytes increase the risk of nodal progression. These previous studies did not characterize tumor-infiltrating lymphocyte subpopulations though, which include cytotoxic $\mathrm{T}$ cells as well as immunosuppressive regulatory $\mathrm{T}$ cells. Ours is the first to examine the predictive value of primary tumor regulatory $\mathrm{T}$ cells for sentinel lymph node positivity, and although our final model of sentinel lymph node positivity risk excludes regulatory $\mathrm{T}$ cells, their presence likely accounts for the observed increased risk of tumorinfiltrating lymphocytes. Regulatory T cell differentiation is promoted by the immunosuppressive cytokine interleukin-10 that also prevents the function of conventional dendritic cells, a dendritic cell subset evaluated for the first time in this study as it 
relates to sentinel lymph node metastasis. The presence of $\mathrm{CD} 11 \mathrm{c}^{+}$conventional dendritic cells protects against nodal progression, which is consistent with findings from a recent study by our group demonstrating that primary melanoma overexpression of miR-30b/30d increases interleukin-10 synthesis by targeting the GalNAc transferase GALNT7. ${ }^{20}$ Interleukin-10 increases primary tumor regulatory $\mathrm{T}$ cells and decreases conventional dendritic cells, and data also support an association between miR-30d and regulatory $\mathrm{T}$ cell recruitment. ${ }^{20}$ Dendritic cells that express CD86 are often regarded as both mature and immunogenic with the capacity to prime $\mathrm{T}$ cells integral to the antitumor immune response, but our study shows that CD86 ${ }^{+}$ dendritic cells increase the risk for sentinel lymph node positivity. These 'mature' dendritic cells may represent tolerogenic indoleamine 2,3-dioxygenase $(\mathrm{IDO})^{+}, \mathrm{CD}_{123^{+}}$plasmacytoid dendritic cells that were found to be associated with poor prognosis in melanoma ${ }^{15}$ rather than immunogenic CD $11 \mathrm{c}^{+}$ conventional dendritic cells that were observed to protect against sentinel lymph node metastasis. CD86 can also be expressed on semi-mature dendritic cells, which are tolerogenic. ${ }^{21}$

The apparent poor prognostic value of $\mathrm{CD}^{+} 6^{+}$ dendritic cells is likewise seen in the comparison of the positive vs negative sentinel lymph node, in which a significantly higher proportion of cells expressing the dendritic cell maturation marker CD86 is found in the positive sentinel lymph node. Data again suggest that these 'mature' dendritic cells are not of the conventional dendritic cell subset as $\mathrm{CD} 11 \mathrm{C}^{+}$cells are significantly decreased in the positive sentinel lymph node compared with the negative sentinel lymph node. The increased number of regulatory $\mathrm{T}$ cells in the positive sentinel lymph node may instead have induced the preferential expansion of $\mathrm{IDO}^{+}, \mathrm{CD}_{123}{ }^{+}$plasmacytoid dendritic cells, ${ }^{22,23}$ as was seen in a breast cancer study where the presence of both Foxp ${ }^{+}$and IDO ${ }^{+}$ cells in the sentinel lymph node accurately discriminated patients with a positive sentinel lymph node from those with a negative sentinel lymph node. ${ }^{24}$ Our results, therefore, provide evidence in support of the proposed positive feedback loop between regulatory $\mathrm{T}$ cells and plasmacytoid dendritic cells where regulatory $\mathrm{T}$ cells induce IDO expression in plasmacytoid dendritic cells via CTLA-4 signaling and IDO-expressing plasmacytoid dendritic cells in turn induce regulatory $\mathrm{T}$ cell differentiation, ${ }^{25}$ which creates a microenvironment conducive to tumor progression. The role of IDOexpressing plasmacytoid dendritic cells in modulating the immunological status of the positive sentinel lymph node is further supported by gene expression data from a melanoma study that showed a trend towards increased IDO levels in the positive sentinel lymph node compared with non-sentinel nodes from the same nodal basin. ${ }^{23}$ Not only do IDO-expressing plasmacytoid dendritic cells downregulate the immune response via regulatory $\mathrm{T}$ cells, but they also cause a decrease in antitumor $\mathrm{T}$ cells. IDO initiates the catabolism of the amino acid tryptophan, whose metabolites induce a stress response in effector T cells resulting in cell-cycle arrest and an increased susceptibility to Fas-mediated apoptosis. ${ }^{26}$

The selective expansion of regulatory $\mathrm{T}$ cells in the positive sentinel lymph node also observed in our study is consistent with findings from a study in breast cancer where the proportion of Foxp $3^{+}$ cells in the sentinel lymph node of patients with regional metastasis was shown to be significantly higher than that of sentinel lymph node-negative patients. ${ }^{24}$ Previous studies evaluating the association between Foxp3 positivity and nodal status in melanoma and lung cancer have similarly shown a significant increase in the frequency of regulatory $\mathrm{T}$ cells in metastatic compared with tumor-free draining lymph nodes. ${ }^{27,28} \mathrm{~A}$ recent study of regional immunity in sentinel lymph nodes with and without melanoma, however, revealed no significant difference in the number of Foxp $3^{+}$cells in the positive sentinel lymph node compared with the negative sentinel lymph node. ${ }^{29}$ Despite the importance of reciprocal tumor-microenvironmental interactions in melanoma progression, these authors excluded areas infiltrated by melanoma cells in their quantification of Foxp3 ${ }^{+}$cells in the positive sentinel lymph node as tumor cells may also stain positive for Foxp $3,^{30}$ an issue addressed in our study as regulatory $\mathrm{T}$ cells were identified by the combination of Foxp3 positivity and characteristic lymphocytic morphology.

Sentinel lymph node positivity delineates a group of melanoma patients at increased risk for recurrence, and most of these patients undergo a completion dissection, which is often followed by systemic adjuvant therapy to lower this risk. Immune markers can provide additional prognostic information as our group has previously identified an immune response gene expression signature that improved the ability of the current American Joint Committee on Cancer melanoma staging system to predict clinical outcome. ${ }^{31}$ In this study, we show that the addition of regulatory $\mathrm{T}$ cell expression in the sentinel lymph node similarly improves the discriminative power of a recurrence risk assessment model based on the American Joint Committee on Cancer stage alone. The immune cell profile of the sentinel lymph node not only provides additional staging information, but it also has the potential to guide adjuvant immunotherapeutic decisions. Adjuvant therapies are associated with considerable toxicity and currently, only interferon$\alpha-2 b^{32}$ is approved by the United States Food and Drug Administration for use in high-risk melanoma patients. Many other systemic adjuvant treatments, such as different types of vaccine approaches or immune modulation and more recently, antiCTLA-4 blockade, have been tested or are currently under investigation. Vaccine antigens are often 
glycosylated, and regulatory $\mathrm{T}$ cells have been shown to downregulate the expression of C-type lectin receptors on dendritic cells that uptake these carbohydrate-bearing antigens. ${ }^{33}$ Regulatory $\mathrm{T}$ cell depletion by biologics like denileukin diftitox and cyclophosphamide would therefore boost the immunological response to the vaccine as would therapeutic agents that inhibit regulatory $\mathrm{T}$ cells. Regulatory $\mathrm{T}$ cells together with IDO-expressing plasmacytoid dendritic cells promote tumor progression by maintaining an immunosuppressive microenvironment. As regulatory $\mathrm{T}$ cells constitutively express CTLA-4 and IDO expression in plasmacytoid dendritic cells is mediated by CTLA-4 signaling, anti-CTLA-4 monoclonal antibodies would effectively break this positive feedback loop, and anti-CTLA-4 monoclonal antibodies like ipilimumab have already demonstrated survival benefit in metastatic melanoma patients ${ }^{34}$ such that it recently received Food and Drug Administration approval for the treatment of unresectable stage III and stage IV melanoma.

In conclusion, our study has shown that the immune profile of the primary melanoma has predictive value for sentinel lymph node positivity and that the addition of primary tumor immune markers as selection criteria for a sentinel lymph node biopsy may aid in the identification of patients with occult nodal metastasis. Data also suggest that the immunological status of the sentinel lymph node, the initial site of metastasis, provides information for both staging and informed therapeutic decisions that can potentially be used to restore an effective antitumor immune response.

\section{Acknowledgement}

This work was supported by the Dr Alfred W Kopf Research Grant Award from The Skin Cancer Foundation (to FD), the National Cancer Institute Cancer Center Support Grant (Grant No. 5 P30 CA 016087-27 to IO), and the Marc Jacobs Campaign to support the Interdisciplinary Melanoma Cooperative Group.

\section{Disclosure/conflict of interest}

The authors declare no conflict of interest.

\section{References}

1 Gershenwald JE, Thompson W, Mansfield PF, et al. Multi-institutional melanoma lymphatic mapping experience: the prognostic value of sentinel lymph node status in 612 stage I or II melanoma patients. J Clin Oncol 1999;17:976-983.

2 Balch CM, Gershenwald JE, Soong SJ, et al. Final version of 2009 AJCC melanoma staging and classification. J Clin Oncol 2009;27:6199-6206.
3 Testori A, De Salvo GL, Montesco MC, et al. Clinical considerations on sentinel node biopsy in melanoma from an Italian multicentric study on 1,313 patients (SOLISM-IMI). Ann Surg Oncol 2009;16:2018-2027.

4 Warycha MA, Zakrzewski J, Ni Q, et al. Meta-analysis of sentinel lymph node positivity in thin melanoma ( $\leq 1 \mathrm{~mm}$ ). Cancer 2009;115:869-879.

5 Kaur C, Thomas RJ, Desai N, et al. The correlation of regression in primary melanoma with sentinel lymph node status. Clin Pathol 2008;61:297-300.

6 Morris KT, Busam KJ, Bero S, et al. Primary cutaneous melanoma with regression does not require a lower threshold for sentinel lymph node biopsy. Ann Surg Oncol 2008;15:316-322.

7 White Jr RL, Ayers GD, Stell VH, et al. Factors predictive of the status of sentinel lymph nodes in melanoma patients from a large multicenter database. Ann Surg Oncol 2011;18:3593-3600.

8 Kruper LL, Spitz FR, Czerniecki BJ, et al. Predicting sentinel node status in AJCC stage I/II primary cutaneous melanoma. Cancer 2006;107:2436-2445.

9 Taylor RC, Patel A, Panageas KS, et al. Tumorinfiltrating lymphocytes predict sentinel lymph node positivity in patients with cutaneous melanoma. J Clin Oncol 2007;25:869-875.

10 Mandalà M, Imberti GL, Piazzalunga D, et al. Clinical and histopathological risk factors to predict sentinel lymph node positivity, disease-free and overall survival in clinical stages I-II AJCC skin melanoma: outcome analysis from a single-institution prospectively collected database. Eur J Cancer 2009;45:2537-2545.

11 Chiba T, Ohtani H, Mizoi T, et al. Intraepithelial CD8+ T-cell-count becomes a prognostic factor after a longer follow-up period in human colorectal carcinoma: possible association with suppression of micrometastasis. Br J Cancer 2004;91:1711-1717.

12 Rauser S, Langer R, Tschernitz S, et al. High number of CD45RO+ tumor infiltrating lymphocytes is an independent prognostic factor in non-metastasized (stage I-IIA) esophageal adenocarcinoma. BMC Cancer 2010; 10:608.

13 Lee HE, Chae SW, Lee YJ, et al. Prognostic implications of type and density of tumour-infiltrating lymphocytes in gastric cancer. Br J Cancer 2008;99:1704-1711.

14 French JD, Weber ZJ, Fretwell DL, et al. Tumor-associated lymphocytes and increased FoxP3+ regulatory $\mathrm{T}$ cell frequency correlate with more aggressive papillary thyroid cancer. J Clin Endocrinol Metab 2010;95:2325-2333.

15 Jensen TO, Schmidt H, Møller HJ, et al. Intratumoral neutrophils and plasmacytoid dendritic cells indicate poor prognosis and are associated with pSTAT3 expression in AJCC stage I/II melanoma. Cancer 2011, (Epub ahead of print).

16 Zitvogel L, Tesniere A, Kroemer G. Cancer despite immunosurveillance: immunoselection and immunosubversion. Nat Rev Immunol 2006;6:715-727.

17 Wich LG, Hamilton HK, Shapiro RL, et al. Developing a multidisciplinary prospective melanoma biospecimen repository to advance translational research. Am J Transl Res 2009;1:35-43.

18 Gromet MA, Epstein WL, Blois MS. The regressing thin malignant melanoma: a distinctive lesion with metastatic potential. Cancer 1978;42:2282-2292.

19 Guitart J, Lowe L, Piepkorn M, et al. Histological characteristics of metastasizing thin melanomas: a case-control study of 43 cases. Arch Dermatol 2002;138:603-608. 
20 Gaziel-Sovran A, Segura MF, Di Micco R, et al. miR$30 \mathrm{~b} / 30 \mathrm{~d}$ regulation of GalNAc transferases enhances invasion and immunosuppression during metastasis. Cancer Cell 2011;20:104-118.

21 Bayry J, Triebel F, Kaveri SV, et al. Human dendritic cells acquire a semimature phenotype and lymph node homing potential through interaction with CD4+ CD25+ regulatory $\mathrm{T}$ cells. J Immunol 2007;178: 4184-4193.

22 Munn DH, Sharma MD, Hou D, et al. Expression of indoleamine 2,3-dioxygenase by plasmacytoid dendritic cells in tumor-draining lymph nodes. J Clin Invest 2004;114:280-290.

23 Lee JH, Torisu-Itakara H, Cochran AJ, et al. Quantitative analysis of melanoma-induced cytokine-mediated immunosuppression in melanoma sentinel nodes. Clin Cancer Res 2005;11:107-112.

24 Mansfield AS, Heikkila PS, Vaara AT, et al. Simultaneous Foxp3 and IDO expression is associated with sentinel lymph node metastases in breast cancer. BMC Cancer 2009;9:231.

25 Mahnke K, Bedke T, Enk AH. Regulatory conversation between antigen presenting cells and regulatory T cells enhance immune suppression. Cell Immunol 2007; 250:1-13.

26 Lee SM, Lee YS, Choi JH, et al. Tryptophan metabolite 3-hydroxyanthranilic acid selectively induces activated $\mathrm{T}$ cell death via intracellular GSH depletion. Immunol Lett 2010;132:53-60.

27 Viguier M, Lemaître F, Verola O, et al. Foxp3 expressing CD4+CD25(high) regulatory $\mathrm{T}$ cells are over- represented in human metastatic melanoma lymph nodes and inhibit the function of infiltrating $\mathrm{T}$ cells. J Immunol 2004;173:1444-1453.

28 Schneider T, Kimpfler S, Warth A, et al. Foxp3+ regulatory $\mathrm{T}$ cells and natural killer cells distinctly infiltrate primary tumors and draining lymph nodes in pulmonary adenocarcinoma. J Thorac Oncol 2011;6: 432-438.

29 Mansfield AS, Holtan SG, Grotz TE, et al. Regional immunity in melanoma: immunosuppressive changes precede nodal metastasis. Mod Pathol 2011;24: $487-494$

30 Ebert LM, Tan BS, Browning J, et al. The regulatory T cell-associated transcription factor FoxP3 is expressed by tumor cells. Cancer Res 2008;68:3001-3009.

31 Bogunovic D, O’Neill DW, Belitskaya-Levy I, et al. Immune profile and mitotic index of metastatic melanoma lesions enhance clinical staging in predicting patient survival. Proc Natl Acad Sci USA 2009;106:20429-20434.

32 Sabel MS, Sondak VK. Pros and cons of adjuvant interferon in the treatment of melanoma. Oncologist 2003;8:451-458.

33 Navarrete AM, Delignat S, Teillaud JL, et al. CD4 ${ }^{+}$ $\mathrm{CD}^{2} 5^{+}$regulatory $\mathrm{T}$ cell-mediated changes in the expression of endocytic receptors and endocytosis process of human dendritic cells. Vaccine 2011;29: 2649-2652.

34 Hodi FS, O’Day SJ, McDermott DF, et al. Improved survival with ipilimumab in patients with metastatic melanoma. N Engl J Med 2010;363:711-723.

Supplementary Information accompanies the paper on Modern Pathology website (http://www.nature.com/ modpathol) 